\title{
Characteristics of electromagnetic Taiwanese-Railway signals and the signals' influences on magnetotelluric measurements
}

\author{
Ping-Yu Chang ${ }^{1,2, *}$, Han-Lun Hsu ${ }^{1}$, Ding-Jun Lin $^{1}$, Azhar Fikri ${ }^{1}$, and Jordi Mahardika Puntu ${ }^{1}$ \\ ${ }^{1}$ Department of Earth Sciences, National Central University, Taoyuan City, Taiwan \\ ${ }^{2}$ Earthquake Disaster \& Risk Evaluation and Assessment Center, Taoyuan City, Taiwan
}

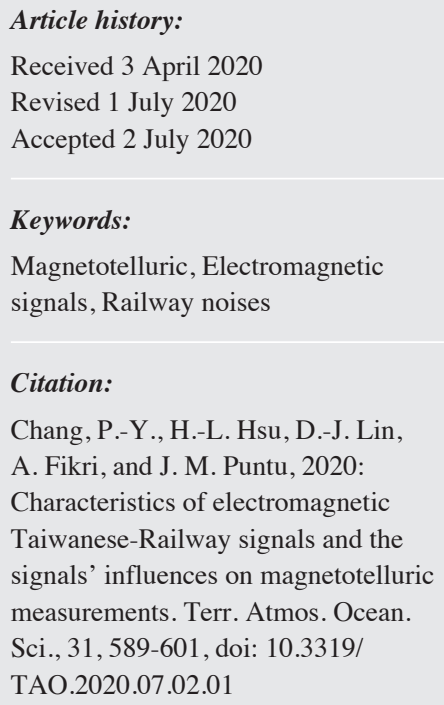

\begin{abstract}
We measured electromagnetic responses near tracks of Taiwan's railway system in order to identify the possible influence of these artificial signals, which derive from several types of electric trains. Our power-spectrum analysis shows that the noise frequencies of the "broadband" train signals extend from $10^{-2}$ to over $10 \mathrm{~Hz}$ after applying the $60-\mathrm{Hz}$ notch filter. We also discovered that different types of trains exhibit unique electromagnetic-signal characteristics. Hence we were able to pinpoint the train-based sources of the signals. If we exclude the train signals from the spectrum measurements, we obtain a consistent estimation of apparent resistivity in relation to our data that we collected in the quiet early morning hours after spectrum normalization for sites that are over $1 \mathrm{~km}$ from train tracks. We also observed that leakage current may induce ULF noise and therefore influence the estimation of apparent resistivity. Sites near tracks suffer from both leakage current and magnetic dipole effects, resulting in inconsistencies between our apparent resistivity estimations and the estimations from quiet periods. Moreover, the train noises exhibit a different diffusion behavior from the natural signals. As a result, one should avoid using measurements that contain train signals. In the study, we did not examine the phase components of the train-contaminated and the noise-free magnetotelluric measurements. Our future work will focus on the phase components of signals as they relate to the influences of electric trains and high-speed railroads for subsurface exploration.
\end{abstract}

\section{INTRODUCTION}

Electromagnetic signals from stationary sources may contribute significant noise to electromagnetic geophysical surveys, such as those involving passive magnetotelluric (MT) methods. In Taiwan the electric railroad system circles the island and may serve as a major source of electromagnetism in the environment. This possibility underscores the importance of analyzing the signals associated with the electric railroad system, with an emphasis on studying the relevance of electromagnetic signals for geophysical explorations. Taiwan's railway system uses overhead power lines with an alternative (AC) power supply of $26 \mathrm{kV} / 60 \mathrm{~Hz}$ for charging the electric trains (e.g., Huang et al. 2001). The entire power-line network in this railway system is divided into independent sectors: 14 in western Taiwan and 5 in eastern Taiwan (Acswoa 2019). The average length of each

\footnotetext{
* Corresponding author

E-mail:pingyuc@ncu.edu.tw
}

power-line sector is about $40 \mathrm{~km}$. There are neutral zones between the two neighboring sectors. Every sector has one power substation that transforms the high-voltage electric power and outputs it from the power grid into the catenary wire system, as shown in Fig. 1. The power travels through the overhead catenary system with one feeding wire over the railroad. The electric trains can raise their pantographs in order to come into contact with the feeding line and can, in this way, acquire electric power for the motors during passage through a given sector. Excess electric current can thus flow through the trains and into steel railroad tracks grounded in the earth.

Electromagnetic signals from electric trains, highspeed railways, and urban metro systems create significant artificial noise that may hamper researchers' efforts to measure the nature of EM responses. Moreover, these signals are "coherent" and are difficult to distinguish from other signals. Without knowing the pattern and magnitude of 
trains' EM responses, researchers can encounter significant stumbling blocks that impede geophysical analysis and that warp interpretations. For instance, researchers usually use remote references in magnetotelluric methods to remove non-coherent noise, yet coherent artificial noise may seriously contaminate data involving remote magnetic signals. Unfortunately, the literature lacks detailed examinations of these electric-train signals, and hence we lack sufficient knowledge for avoidance of coherent noise's unwanted influence on local magnetotelluric measurements.

Electric-current leakage into the ground may directly affect magnetotelluric measurements over tens of kilometers (e.g., Pádua et al. 2002; Tanbo et al. 2003), and may also give rise to ultra-low frequency (ULF) fields that look like pulse signals in time domains (e.g., Jones and Kelly 1966; Fraser-Smith 1981; Egbert et al. 2000). The ULF fields induced by leaking current may sometimes inject noise into such sensors as broad-band vertical seismic sensors placed near train tracks (Forbriger 2007). Szarka (1988) reviewed the extensive literature on "cultural" (i.e., human-made) electromagnetic signals including those induced by electric trains. Lowes (2009) used several models for calculating the relationships between the distance to the track and the induced magnetic fields due to current leakage.

Larsen et al. (1994) stated that electric-train systems possibly generate two modes of noise contamination: one mode is due to electric currents leaking through grounded train tracks; the other mode is due to the magnetic dipole effect resulting from a flow of currents through overhead power lines to the tracks. Linington (1974) proposed that sites more than $1 \mathrm{~km}$ from tracks are unlikely to be affected by the magnetic dipole effect, since the magnetic dipole effect decays rapidly with distance. Leakage current, thus, is a major contributor of "cultural" electromagnetic noise to MT measurements.

In order to identify the electromagnetic signals that are associated with electric trains, we deployed MT stations near the railway tracks between the Lodung and Dongshan stations in northeastern Taiwan's Ilan County. The northeastern part of Taiwan, being less populated than the western stretch of the island, has a railway system that is far less busy than the one out west. Therefore, researchers would do well to conduct in-depth investigations of this train-based noise. Our present study focuses on two main issues regarding the noise: (1) analyzing the spectrum and orientation of noise associated with the train system and (2) evaluating the train noise's influence on both MT-impedance estimation and apparent resistivity.

\section{INSTRUMENTS AND MEASUREMENT CONFIGURATIONS}

For collecting the electromagnetic responses, we used $\mathrm{Pb}-\mathrm{PbSO}_{4}$ non-polarized electrodes to measure the telluric field, and the Fluxgate magnetometer, the Bartington ${ }^{\mathrm{TM}}$ Mag-03, to measure the magnetic field. The Mag-03 is a three-axis magnetic field sensor that has resolution up to 6 pT and a wide frequency response band extending from DC to $3 \mathrm{kHz}$. To record the electric and magnetic field measurements, we used the Earth Data ${ }^{\mathrm{TM}}$ EDR-210, which is a datalogger capable of providing 6 independent channels for recording measurements with GPS timing and has high dynamic range with a true 24-bit A/D convertor. The sampling rate of the EDR-210 is between 1 and $3000 \mathrm{~Hz}$ and is therefore suitable for collecting the Mag-03's magnetic measurements. The EDR-210 can store field measurements
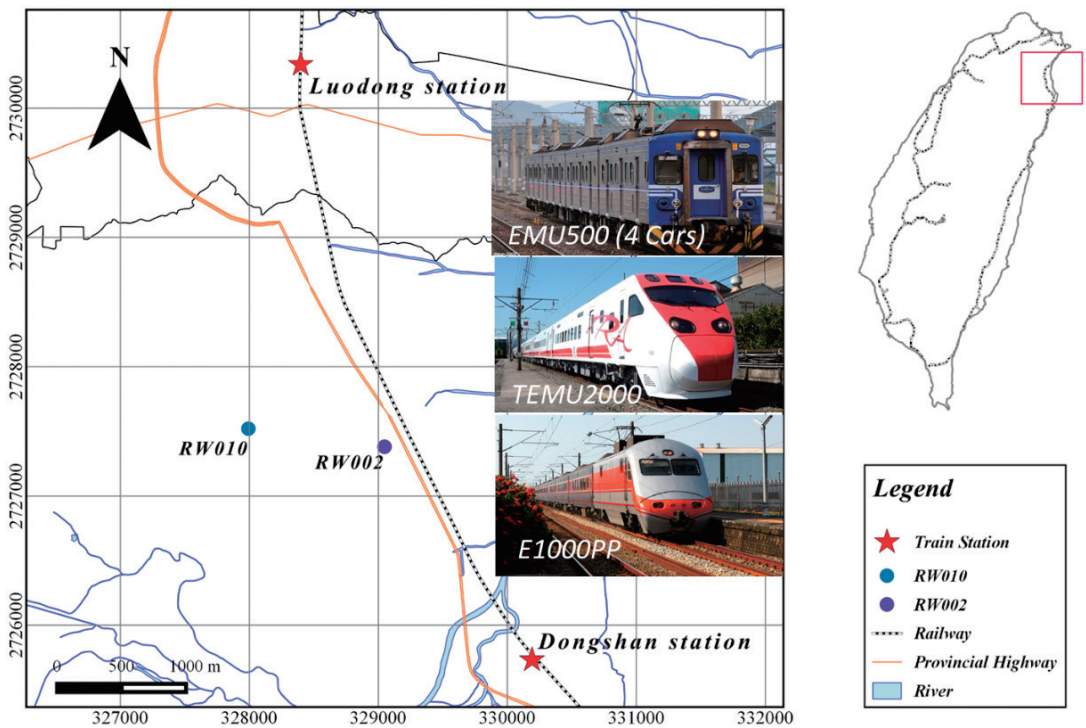

Fig. 1. The locations of the MT stations (red stars) and the train tracks (the yellow line) (edited from https://zh.wikipedia.org/wiki/\%E5\%8F\%B0\% E7\%81\%A3\%E9\%90\%B5\%E8\%B7\%AF). 
either on a CF memory card or in an SSD hard drive using MiniSEED or ASC II formats.

In our study, the area between the Lodung and Dongshan stations was the site of three MT stations (as shown in Fig. 1) and, being less populated than other potential areas of measurement, was the optimal site for our study. The MT stations were deployed roughly perpendicular to the railway tracks at distances of $200 \mathrm{~m}$ and $1.1 \mathrm{~km}$. Data from the MT stations were synchronized with the data logger's built-in GPS clock. Using a sampling rate of $500 \mathrm{~Hz}$, we collected the responses of the paired electric and magnetic fields for 12 consecutive hours (from 20:00 on 21 December to 08:00 on 22 December 2017). This data-collection time extended from a period when the trains were operating to a period in the early morning, when no trains were passing. In this way, we sought to identify the artificial signals from the electrictrain system and to analyze their spectral responses.

\section{RESULTS}

\subsection{The Waveform and Spectra of the Observed Electric and Magnetic Fields}

Figure 2 shows the electric-field time-series that is parallel to the tracks (Ex) and the magnetic-field time-series that is perpendicular to the tracks (Hy), with all data collected between 22:00 and 22:59 at Station RW002. The strength of the electrical-field Ex falls mostly between +250 and $-250 \mathrm{mV} \mathrm{km}^{-1}$, except for several spikes that exceed this range. Because the electric trains in Taiwan operate at 60 $\mathrm{Hz}$ AC power, the measured signals show a symmetric AC pattern in the raw time-series of the Ex field's strength. The strength of the magnetic-field Hy ranges between +10 and $-10 \mathrm{nT}$. In contrast to the Ex signals, we observed six sharp spikes with absolute amplitude over $20 \mathrm{nT}$. Between 22:00 and 22:59 on 21 December, six trains passed through the observation site (Table 1): the express Puyuma PYM-252, the express Tzichiang TC-251, the commuter Local-420, the express Tzichiang TC-253, the express Puyuma PYM-288, and the express Puyuma PYM-447, all of which were travelling between Dongshan and Luodong (TRA 2017). The aforementioned magnetic spikes stem from these trains' operations, as the timetable in Table 1 shows.

We examined the spectrum and time-series waveforms of the collected electric and magnetic signals by applying the power-line filtering to the data collected between 22:00 and 22:59 at the RW002 station. After the power-line filtering, we observed that most of the Ex field's strength dropped below $10 \mathrm{mV} \mathrm{km}^{-1}$ (Fig. 3). We found six spikes with field strengths higher than $150 \mathrm{mV} \mathrm{km}^{-1}$ in the filtered Ex time-series. The spikes are consistent with the corresponding period's train timetable (see Fig. 3). The filtered Hy fields show that the magnetic fields are lower than $5 \mathrm{nT}$ in general after the power-line filtering is accounted for. We noted that six spikes with a magnetic strength over $-15 \mathrm{nT}$ were consistent with the train schedule. The findings suggest that the electric trains have the most influence (1) on the electric field in the direction parallel to the train track and (2) on the magnetic field in the direction perpendicular to the train track. Curiously, the filtered Ey field does not exhibit the peaks of the electric-train signals, in contrast to the Ex field. The Hx field varies between +5 and $-5 \mathrm{nT}$ and presents peaks that are smaller than $5 \mathrm{nT}$ in magnitude. In general, the findings show that, even though the station is only $200 \mathrm{~m}$ from the train tracks, the electric field perpendicular to the train tracks and the magnetic field parallel to the train tracks are much less sensitive to the trains' influences than their orthogonal field pairs.

Figure 4 presents the time-spectrum diagram, which calculates the power spectral density (PSD) within a 3-minute period for the data collected between 22:00 and 22:59

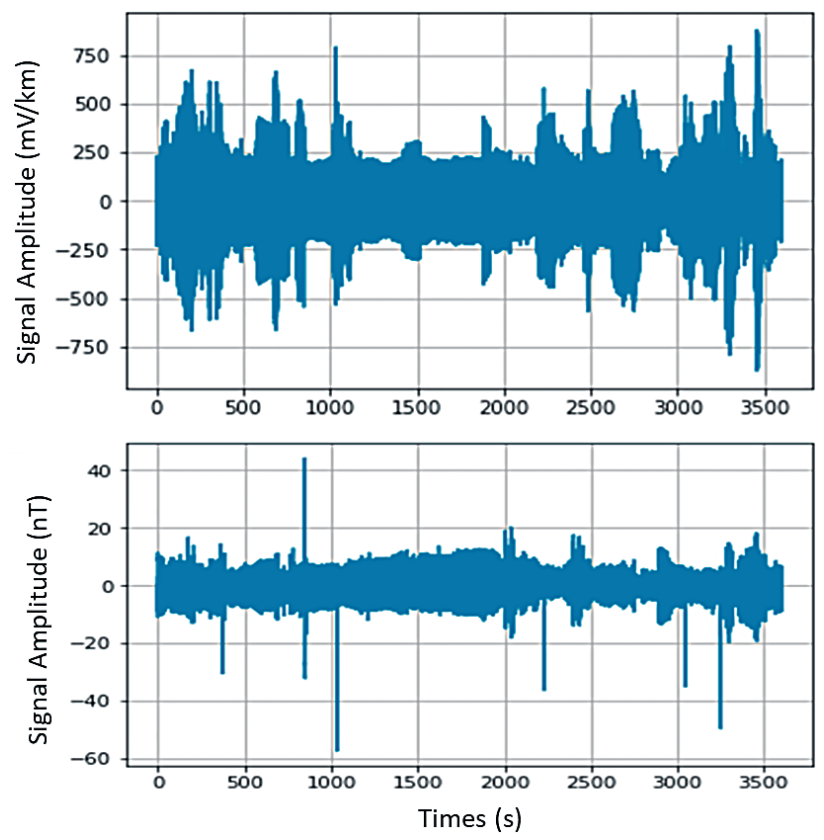

Fig. 2. The time series of the electric field perpendicular to the track (Ex) and the magnetic field parallel to the track $(\mathrm{Hy})$ collected between 22:00 and 22:59 on 12/21/17 at the RW002 station (without filtering).

Table 1. The Luodong station timetable for the 21:50 - 23:00 period on $12 / 21 / 17$.

\begin{tabular}{cccc}
\hline Train \# & Time & Bound & Train Type \\
\hline Local-4247 & $21: 56$ & North & EMU500, 4 Cars \\
PYM-252 & $22: 07$ & South & TEMU2000, 8 cars \\
TC-251 & $22: 14$ & North & E1000-PP, 12 Cars \\
Local-4240 & $22: 15$ & South & EMU500, 8 Cars (4 + 4 Cars) \\
TC-253 & $22: 32$ & North & E1000-PP, 12 Cars \\
PYM-288 & $22: 41$ & South & TEMU2000, 8 cars \\
PYM-447 & $22: 52$ & North & TEMU2000, 8 cars \\
\hline
\end{tabular}



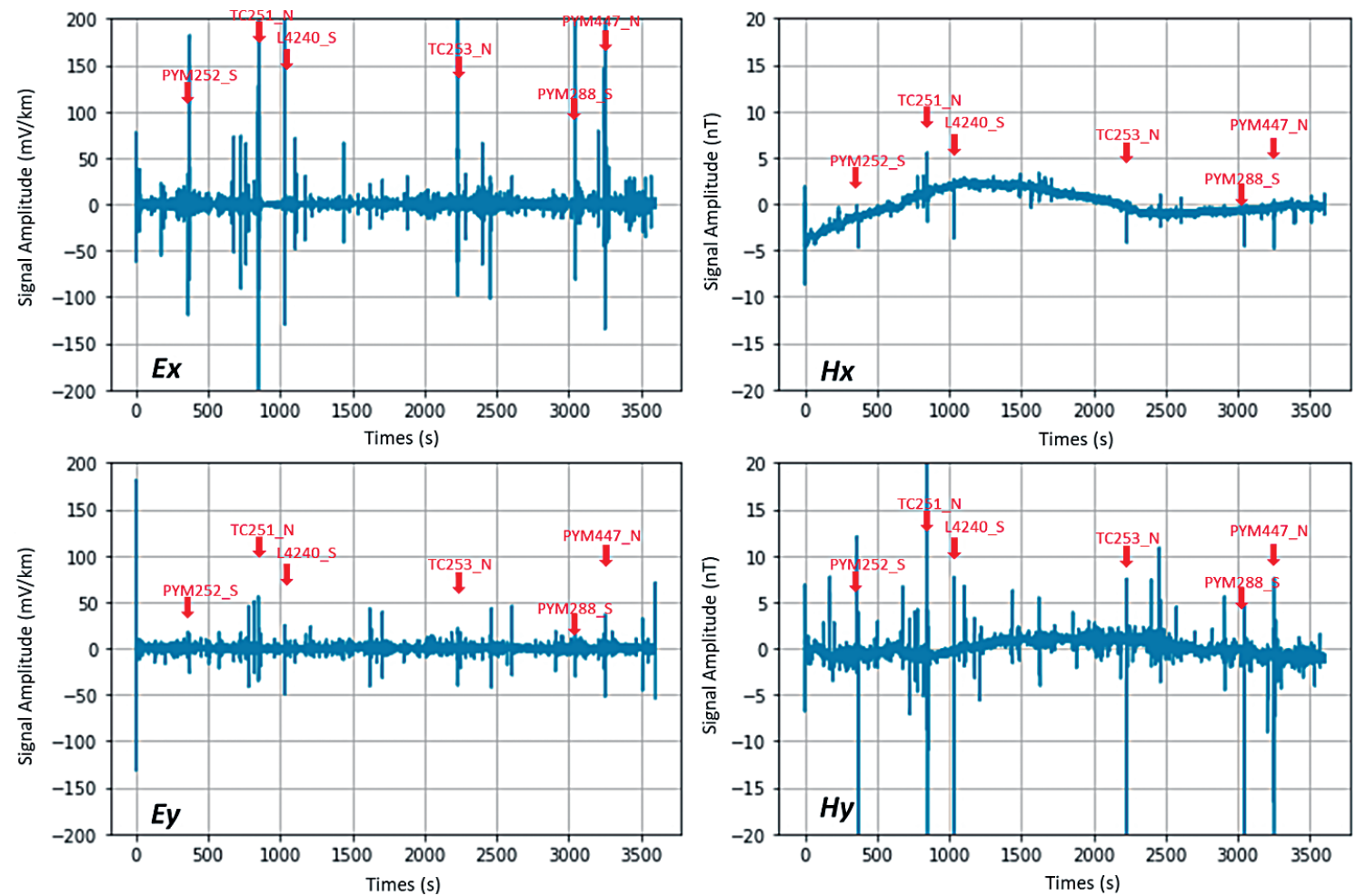

Fig. 3. The time series of the electric fields (Ex and Ey) and magnetic fields (Hx and Hy) collected between 22:00 and 22:59 on 12/21/17 at the RW002 station (after application of a power-line filter; the red arrows indicate the times of the trains' passing).
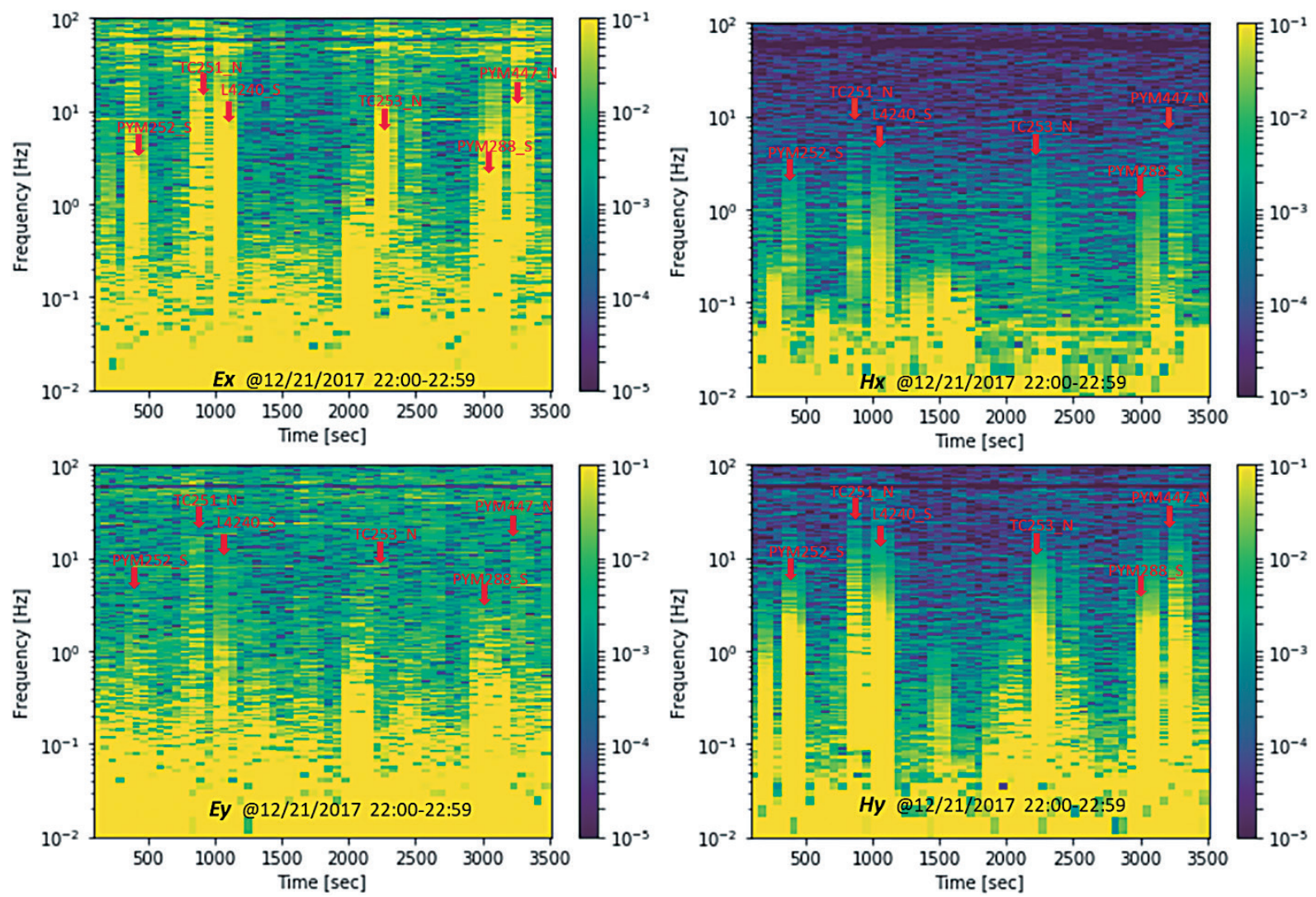

Fig. 4. The frequency-time spectrum maps of the electric and magnetic fields collected between 22:00 and 22:59 on 12/21/17 at the RW002 station. 
at the RW002 site on 21 December 2017. We observed that the "broadband" signal spectra extending from $10^{-2}$ to over $10^{2} \mathrm{~Hz}$ with a PSD higher than $10^{-1} \mathrm{mV}^{2} \mathrm{~Hz}^{-1}$ and $10^{-2} \mathrm{nT}^{2} \mathrm{~Hz}^{-1}$ for Ex and Hy, respectively, appear when electric trains pass through the region between the Lodung and Dongshan stations. By contrast, the background PSD of the electric fields varies only between $10^{-2}$ and $10^{-3} \mathrm{mV}^{2} \mathrm{~Hz}^{-1}$; likewise, the PSD of the magnetic fields varies only between $10^{-2}$ and $10^{-3} \mathrm{nT}^{2} \mathrm{~Hz}^{-1}$. We also observed some broadband signals showing up at the same time in the Ex and Hy fields, but with less PSD than was with the train noise.

Figure 5 shows the $60 \mathrm{~Hz}$-notch-filtered time-series diagram of the electric- and magnetic-field signals collected between 22:00 and 22:59 on 21 December 2017, at the RW010 site, about $1.1 \mathrm{~km}$ away from the train tracks. Most of the amplitudes of the electric-field strength vary between +15 and $-15 \mathrm{mV} \mathrm{km}^{-1}$. We observed several peaks exceeding $30 \mathrm{mV} \mathrm{km}^{-1}$ in the time-series diagram of the Ex field. These peaks correlate to the schedule of the electric trains operating in the area during the same period. However, we observed other peaks with a strength over $\pm 20 \mathrm{mV} \mathrm{km}^{-1}$ in the filtered Ex-field diagram, and they may suggest local artifact noise other than the noise created by electric trains. The Ey-field diagram shows that the time-series variations fall within $\pm 20 \mathrm{mV} \mathrm{km}^{-1}$ with only one signal peak exceeding the variation range at around 2200 seconds. The peak is not associated with the train schedule and is likely from local artifacts. By contrast, the magnetic fields present obvious peaks with an absolute magnitude slightly over 1.5 and $2.5 \mathrm{nT}$ for the Hx and Hy time-series, respectively. These signal peaks are consistent with the times at which electric trains passed through the area during the measurement period.

The time-spectrum diagram in Fig. 6 shows the time variations of the PSD of different frequencies for the data collected between 22:00 and 22:59 at the RW010 site. The diagram shows that, in the Ex, Hx, and Hy spectrograms, the "broadband" signal spectrum extending from $10^{-2}$ to over $10 \mathrm{~Hz}$ is present for the times when electric trains passed through the region (see Fig. 6). The Ey field, however, does not show a distinct response to the electric trains' presence and thus, in this regard, differs from the Ex, $\mathrm{Hx}$, and $\mathrm{Hy}$ fields. Compared with the spectra of train signals in Fig. 4, the PSD of the train signals at RW010 is attenuated to the background level for frequencies over $10 \mathrm{~Hz}$. We also noted that additional strong signals with a frequency of 20 and $10 \mathrm{~Hz}$ appear from 1400 to 2200 seconds and from 2200 to 3600 seconds, respectively, in Fig. 6. These signals may suggest possible human-made noises near the RW010 site.

We compared the signals recorded during the train-operating hours with the signals recorded during the no-trainoperating hours. Figure 7 is the filtered time-series collected at the RW010 site between 02:00 and 02:59 on 22 December 2017. During this period, no passenger trains ran in the region. The magnetic fields, $\mathrm{Hx}$ and $\mathrm{Hy}$, have none of the obvious signal peaks that we observed for these fields during the train-operating hours. However, the electric fields Ex and Ey still exhibit several signal peaks in the range of $\pm 30 \mathrm{mV} \mathrm{km}^{-1}$. Unlike the train signals, these peaks seem to have happened with a certain frequency. This pattern may imply some natural or human-made electromagnetic signals with specific frequencies. We will discuss this point further in the discussion section.

Figure 8 is the time-spectrum PSD diagram collected between 02:00 and 02:59 at the RW010 site. The diagram shows a quieter period, with no obvious train signals in the Ex, Ey, Hx, and Hy fields. In addition, we noted a constant signal possessing a frequency of $10 \mathrm{~Hz}$ during the period between 02:00 and 02:59 on 22 December 2017.

\subsection{Train-Noise Analysis}

To further analyze the train noises and their influences, we focused on only the Ex and Hy pairs, which were affected by the train noises the most, at RW002 and RW010 only in the spectrum analysis. Because no electric train operated during the period between 02:00 and 02:59, the time period has far less train-related EM noise than is the case for the period between 22:00 and 22:59. Hence the measurements collected between 02:00 and 02:59 serve as this study's natural background. From the data presented in Fig. 6, we concluded that six trains had passed through RW002 and RW010 at 390, 870, 1050, 2190, 3090, and 3270 seconds after 22:00. For our train-noise analysis, we obtained the spectra of three-minute time slices that correspond to the times when trains had passed through the sites between 22:00 and 22:59. We also obtained the spectra from the time slices when no trains had passed through the two sites between 22:00 and 22:59. We then calculated the average of the spectra associated with "passing trains" and "no passing trains" between 22:00 and 22:59. Afterward, we compared these two datasets with the natural background for the 02:00 - 02:59 period.

Figure 9 presents two sets of data: first, the average power spectra of electric and magnetic fields with and without train interference for the time segments during the 22:00 - 22:59 period for the RW002 site; and second, the average power spectra of electric and magnetic fields collected during the 02:00 - 02:59 period for the RW002 site. We used the median filter in order to reveal trends and selected 20 frequencies per decade in order to reduce the random peak outliner of the spectra in Fig. 9. This particular site is only about $200 \mathrm{~m}$ away from the train tracks. The power spectra of the train signals, corresponding to EX2200 and HY2200 collected during the 22:00 - 22:59 period, are much greater than the power spectra of EX0200 and HY0200 during the 02:00 - 02:59 period. The power spectra of the train-influenced Ex field, EX2200, is about twenty times greater than the power spectra of the EX2200N, which excludes the train signals during the 22:00 - 22:59 period. The background 

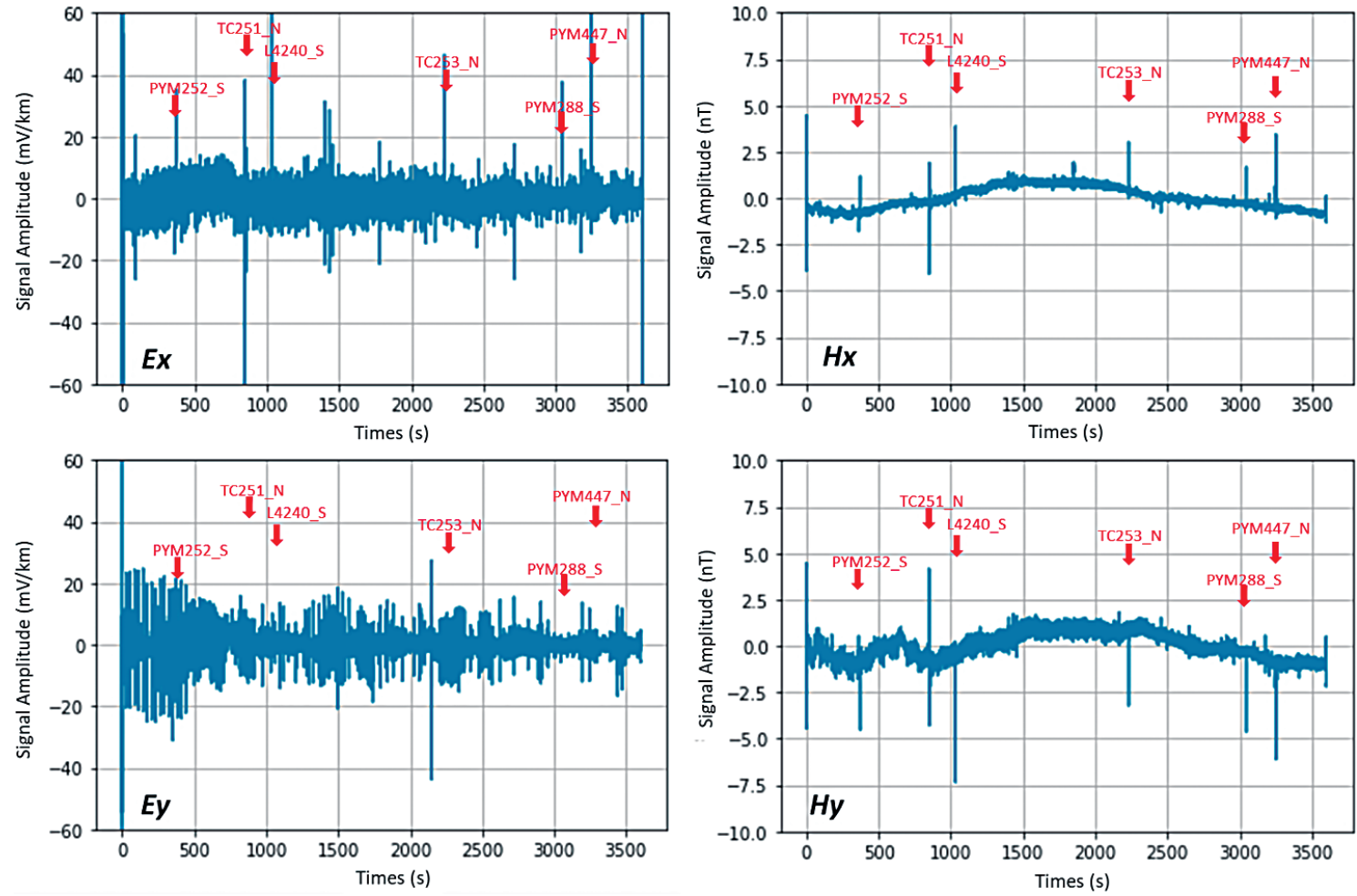

Fig. 5. The time series of the electric fields and magnetic fields collected between 22:00 and 22:59 on 12/21/17 at the RW010 station (after application of a power-line filter).
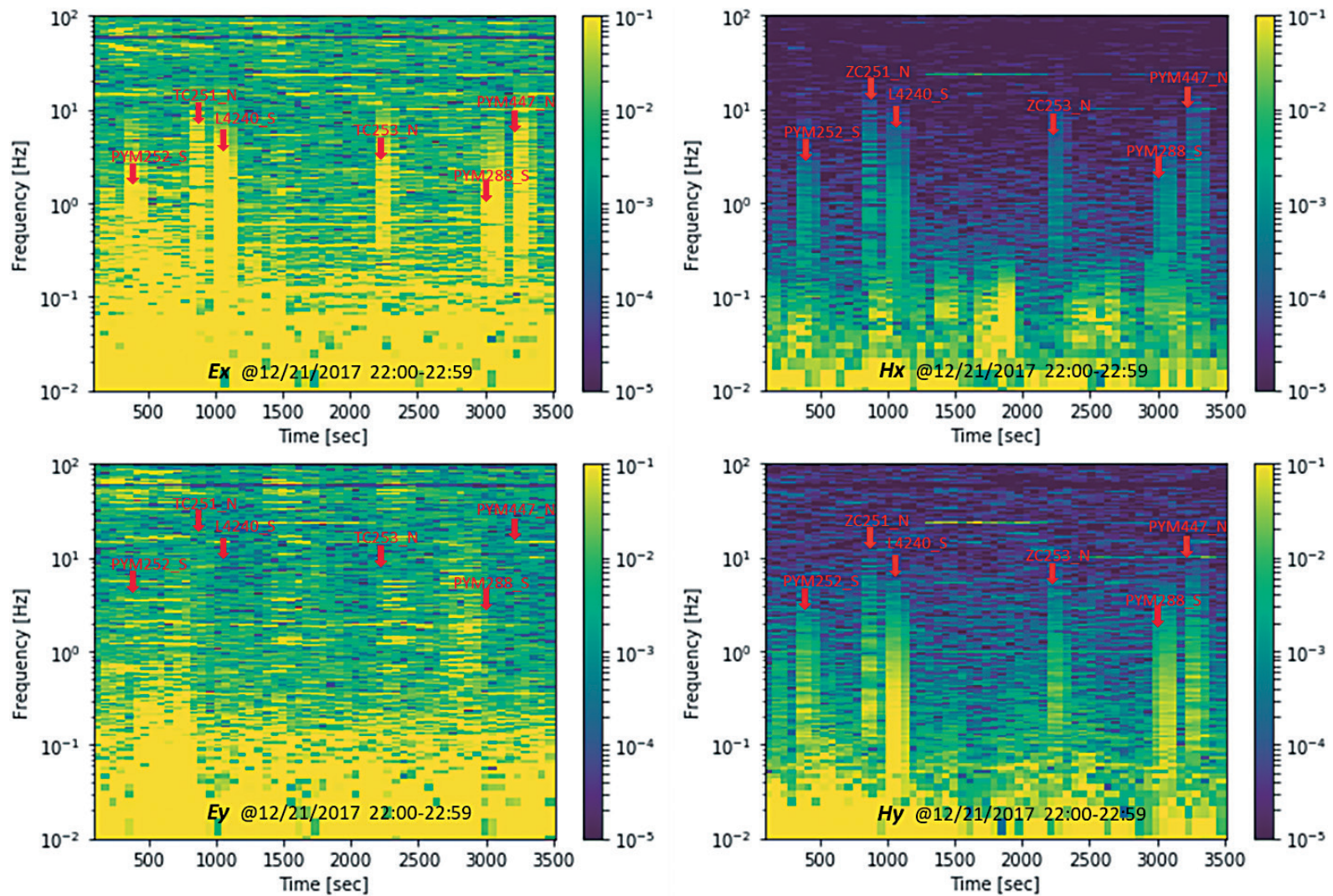

Fig. 6. The frequency-time spectrum maps of the electric and magnetic fields collected between 22:00 and 22:59 on 12/21/17 at the RW010 station. 

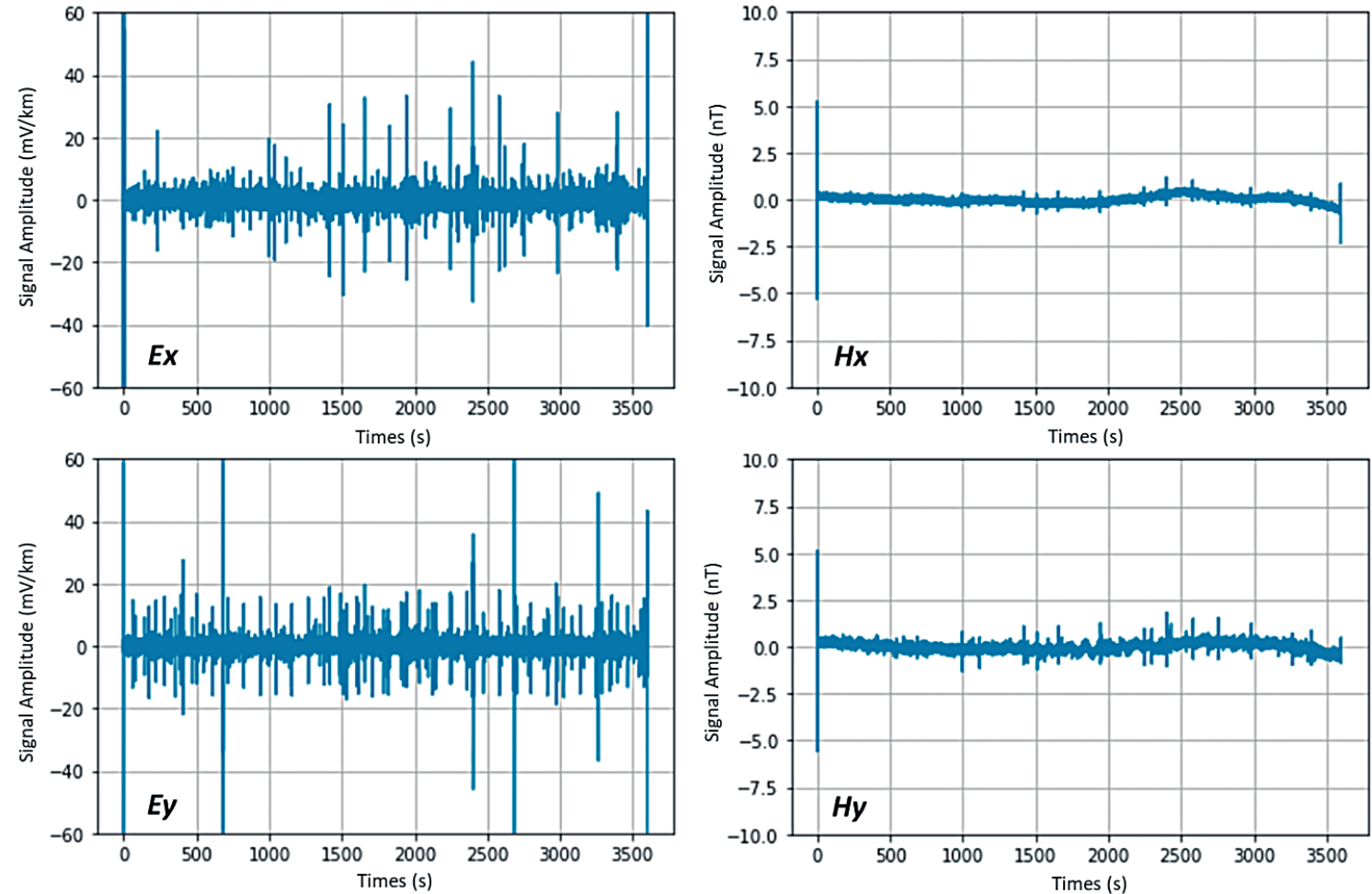

Fig. 7. The time series of the electric fields and magnetic fields collected between 02:00 and 02:59 on 12/22/17 at the RW010 station (after application of a power-line filter).
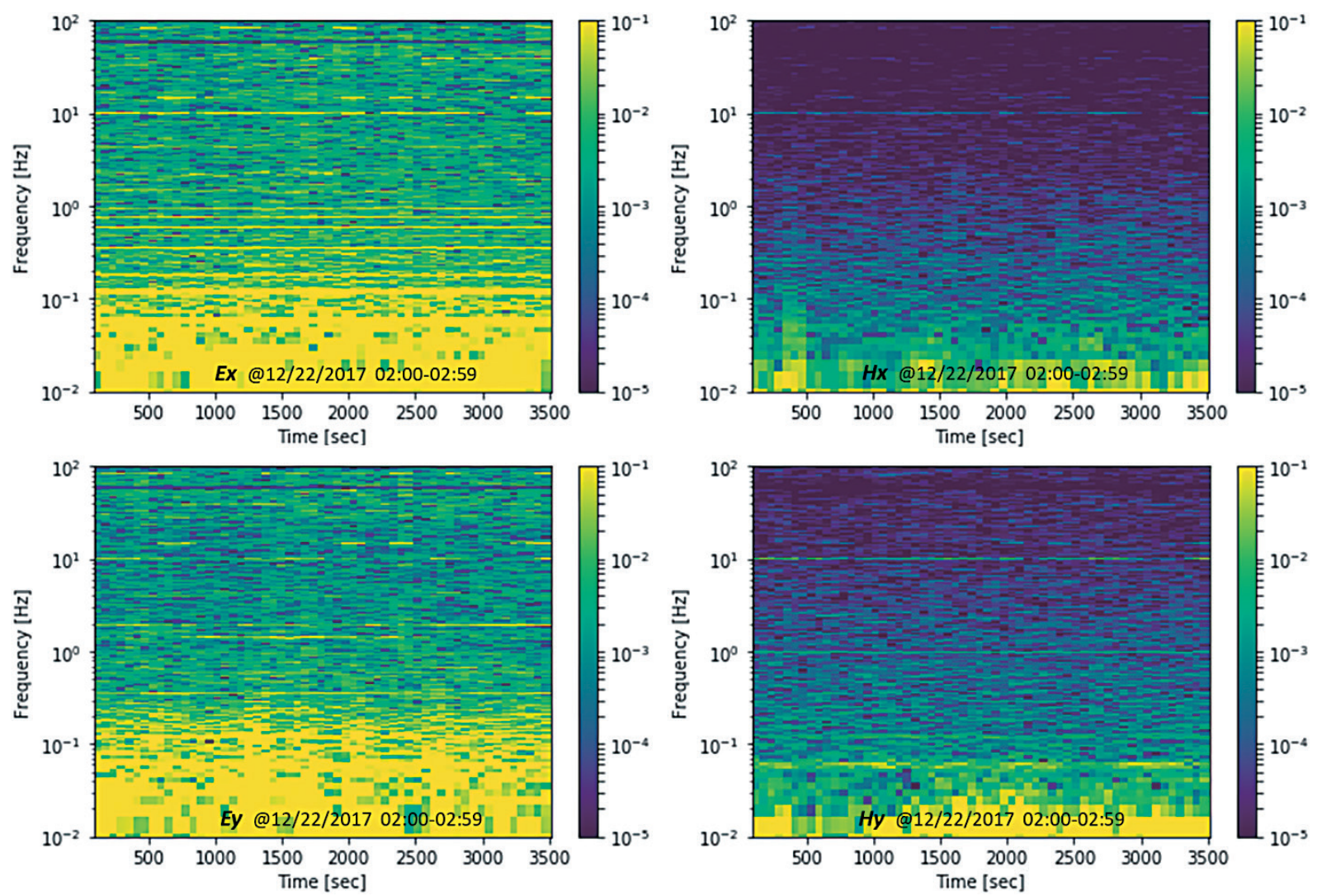

Fig. 8. The frequency-time spectrum maps of the electric and magnetic fields collected between 02:00 and 02:59 on 12/22/17 at the RW010 station. 


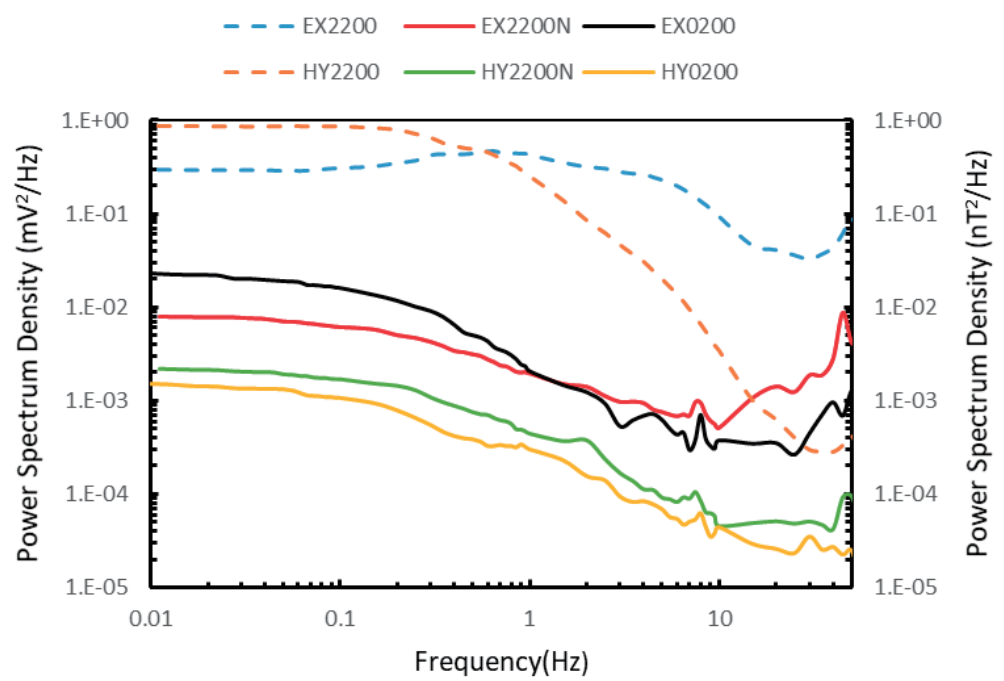

Fig. 9. The RW002 power spectra of the electric and magnetic fields collected during the 22:00 - 22:59 period on 12/21/17 for both the "only trains passing" contexts (EX2200 and HY2200) and the "no trains passing" contexts (EX2200N and HY2200N), and the RW002 power spectra of the background electric and magnetic fields collected during the 02:00-02:59 period on 12/22/17 (EX0200 and HY0200).

power-spectrum curves of the electric fields for EX0200 are about the same range as those for EX2200N. By contrast, the train-influenced magnetic field of HY2200 exhibits a power spectrum that is almost 3 orders of magnitude greater than the magnetic field of HY0200 and HY2200N in the low frequency, and is about an order of magnitude greater than those spectra of HY0200 and HY2200N in the high frequency.

Figure 10 presents two similar basic sets of data as those in RW002: first, the average Ex and Hy spectra collected during the 22:00 - 22:59 period at the RW010 site with and without train influences, and second, the average Ex and Hy spectra collected during the 02:00 - 02:59 period at the same site without any train influences. In Fig. 10 , we have selected 20 frequencies per decade after applying the median filter to represent the distribution of the corresponding apparent resistivity. Both the power spectra of the signals collected without train influences during the 22:00 - 22:59 period and the power spectra of the natural background during the 02:00 - 02:59 period lie within a similar range. The power-spectrum of the electric fields for EX2200N exhibit a similar variation trend to that of the magnetic field for HY2200N. The electric field of EX0200 also shows the similar trend to the magnetic field of HY0200, as can be observed in Fig. 10. The findings suggest that we may be able to acquire good-quality electric and magnetic spectrum data if we can exclude train noises from measurements for sites that are farther than $1 \mathrm{~km}$ from the railway. On the other hand, the power spectra of the electric field EX2200, which involve train noises between 22:00 and 22:59, are about 20 to 30 times greater than the power spectra of EX2200N and EX0200. And the power spectra of the magnetic field HY2200 are about 50 times greater than the power spectra of HY2200N and HY0200, regarding com- ponents with frequencies lower than $1 \mathrm{~Hz}$. Greater than 1 $\mathrm{Hz}$, the power spectra of HY2200 influenced by the train noises gradually reduced to a range similar to the one associated with HY2200N and HY0200. These findings suggest that if one can properly filter out the train signals from the time-spectrum data of the noisy period, the results will be satisfactory and as good as those collected during the quiet "midnight hour".

\section{DISCUSSION}

Comparing Figs. 9 with 10, we observed that the spectra of the HY2200 train-influenced magnetic field at the RW002 site are about two orders of magnitude larger than the corresponding spectra at the RW010 site for frequencies less than $1 \mathrm{~Hz}$, and the difference between the power spectra at RW002 and those at RW010 gradually reduced to about a single order of magnitude in their high-frequency components. By contrast, the power spectra of the EX2200 train-influenced electric field at the RW002 site are about the same as the corresponding power spectra at the RW010 site. According to these findings, the distance from the observation site to the railway tracks seems to have a connection to a train-associated magnetic field that attenuates more rapidly than the train-associated electric field. This rapidly attenuated magnetic field may be affected by the magnetic dipole effect, as predicted in Larsen et al. (1994), from currents flowing through overhead power lines and returning through the tracks. This effect makes sense insofar as the RW002 site is only $200 \mathrm{~m}$ away from the tracks and insofar as Larsen et al. (1994) concluded that the magnetic dipole effect should be insignificant for stations farther than $1 \mathrm{~km}$ from the railway tracks. 


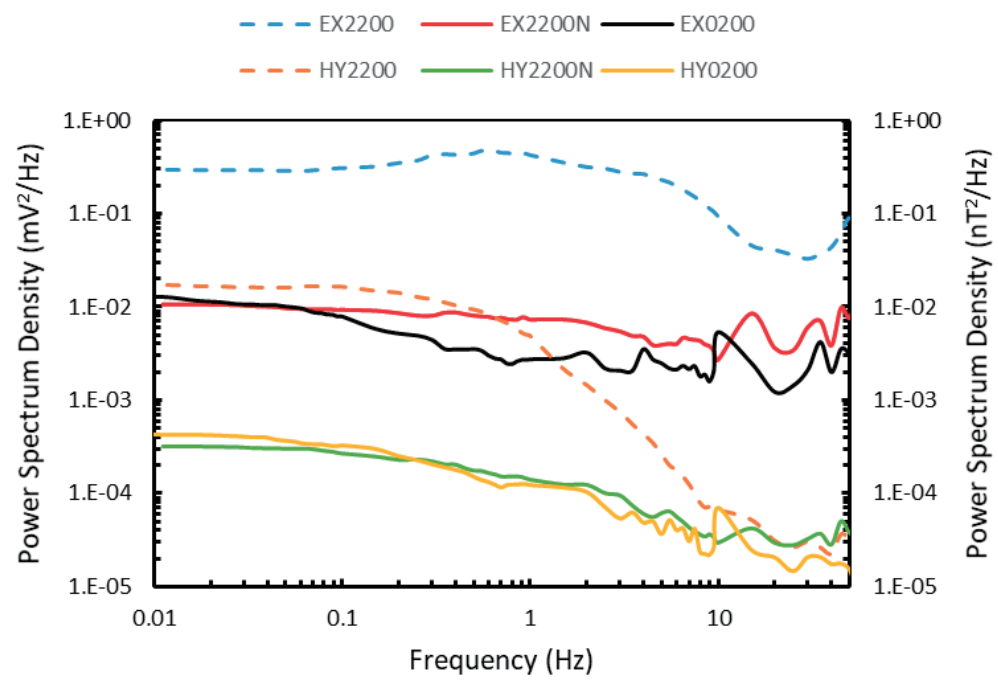

Fig. 10. The RW010 power spectra of the electric and magnetic fields collected during the 22:00-22:59 period on 12/21/17 for both the "only trains passing" contexts (EX2200 and HY2200) and the "no trains passing" contexts (EX2200N and HY2200N), and the RW010 power spectra of the background fields collected during the 02:00 - 02:59 period on 12/22/17 (EX0200 and HY0200).

We calculated apparent resistivity by using conventional one-dimensional far-field estimation. In the study we considered only the Ex-Hy field pairs, since they responded the most to the train influences. The near-field estimation works for the distance $\mathrm{r}$ when $|\mathrm{kr}|>1$ and when wave number $k=\sqrt{i \mu_{0} \omega \sigma}$.

Apparent resistivity can be estimated as

$\rho_{s}=\frac{1}{\mu_{0} \omega}\left|\frac{E_{x}}{H_{y}}\right|^{2}$

where $\mu_{0}$ is magnetic susceptibility in free space, and $\omega$ is angular frequency (Simpson and Bahr 2005). And Cagniard resistivity can be estimated using the following equation:

$\rho_{s}=\frac{1}{5 f} \frac{\left|E_{x}\right|^{2}}{\left|H_{y}\right|^{2}}$

where $f$ is the frequency (Cagniard 1953).

Figure 11a illustrates the calculated apparent resistivities of RHO_2200 (with trains passing only), RHO_2200N (with no trains passing) during the 22:00 - 22:59 period at site RW002. And RHO_0200 in Fig. 11a presents the apparent resistivity calculated with the data collected during the natural background period between 02:00 and 02:59. The trend of RHO_2200 is significantly different from the trends of the other two data sets. This fact implies that train noise has a noteworthy influence on the magnetotelluric measurements at the RW002 site. The apparent resistivities of RHO_2200 and RHO_2200N differ significantly from the resistivity of RHO_0200, although they exhibit the same trend as the RHO_0200 when frequencies are less than $0.3 \mathrm{~Hz}$. Above 1
Hz, the apparent resistivities of RHO_2200 and RHO_2200N exhibit trends completely different from the resistivity of RHO_0200. The magnetic-field's deviation from the trainnoise free measurements to the measurements with only train signals is much greater than the corresponding electricfield's deviation at the RW002 site, resulting in our underestimation of the apparent resistivity of RHO_2200. We also calculated the correlation coefficient for the corresponding Ex-Hy field pairs by applying a 10-datapoint window to the spectra measurements. Figure 11b shows, at the RW002 site, the variations of the correlation coefficients for CORR_2200 (with only train influences during the 22:00 - 22:59 period), for CORR_2200N (with no train influences during the 22:00 - 22:59 period), and for CORR_0200 (the background of the 02:00 - 02:59 period). The "train noise" correlation coefficient, CORR_2200, is negative at low frequencies but is highly positive (between 0.8 and 1 ) when the frequencies are greater than $0.3 \mathrm{~Hz}$. By contrast, the correlation coefficients of CORR_2200N and CORR_0200 are highly positive at low frequencies but slightly positive (less than 0.8 ) for frequencies higher than $1 \mathrm{~Hz}$. These facts suggest that the diffusion mechanism of train noises differs from the diffusion mechanism of natural signals and may degrade estimates of apparent resistivity at low frequencies if one does not properly exclude the train noises from the measurements. Random regional noises may dominate background measurements and measurements excluding train influences for frequencies higher than $1 \mathrm{~Hz}$, since these measurements exhibit low correlations between the spectra of Ex and Hy field pairs.

Figure 11c compares the apparent resistivities of RHO_2200 and RHO_2200N with the background resistivities of RHO_0200 for frequencies less than $1 \mathrm{~Hz}$. Both the resistivity of RHO_2200 and the resistivity of RHO_2200N 

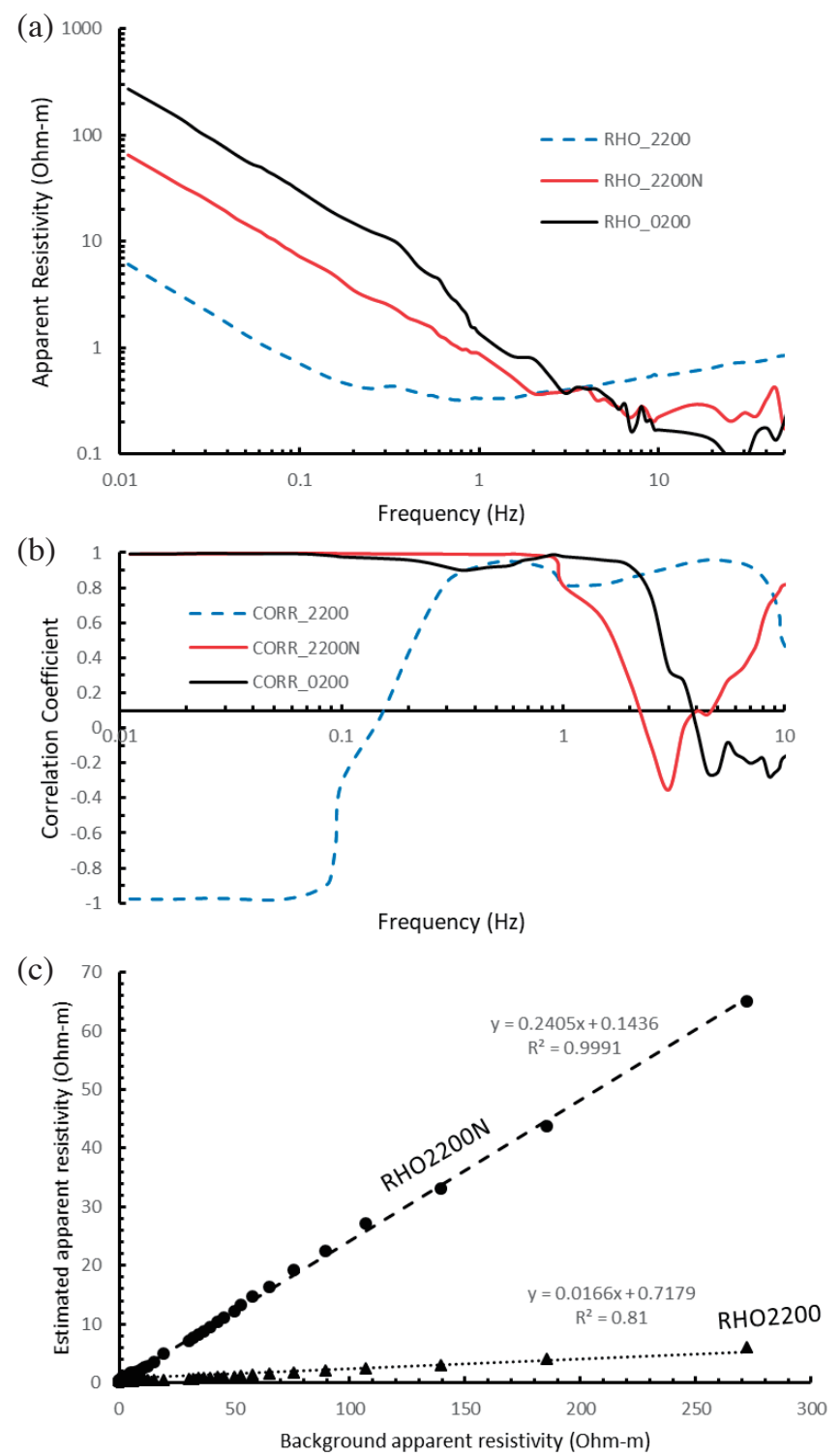

Fig. 11. (a) The RW002 apparent-resistivity measurements collected during the 22:00 - 22:59 period on 12/21/17 for both the "only trains passing" context (RHO_2200) and the "no trains passing" context (RHO_2200N), and the background-resistivity measurements collected during the 02:00 - 02:59 period on 12/22/17 (RHO_0200). (b) The correlation coefficients of the electric and magnetic spectra for the "only trains passing" context (CORR_2200) during the 22:00 - 22:59 period, the "no trains passing" context (CORR_2200N) during the 22:00 - 22:59 period, and the background context (CORR_0200) during the 02:00 - 02:59 period at RW002 on 12/22/17. (c) A comparison of the apparent resistivities of RHO_2200 and RHO_2200N with the background resistivities of RHO_0200 at the RW010 site for frequencies less than 1Hz.

show a good linear correlation to the background resistivity, and their $r^{2}$ values are 0.99 and 0.81 , respectively. The apparent resistivity of RHO_2200 is about 1.6 percent of the background resistivity of RHO_0200. In addition, the apparent resistivity of RHO_2200N is about 24 percent of the background apparent resistivity of RHO_0200.

Figure 12a shows the estimated apparent resistivity of RHO_2200, RHO_2200N, and RHO_0200 at the RW010. The apparent resistivity of RHO_2200N, which involves no train signals during the 22:00 - 22:59 period, is similar to that of the natural background, RHO_0200. In addition, the apparent resistivity of RHO_2200, which involves train noises, is similar to that of RHO_0200 for frequencies less than $1 \mathrm{~Hz}$. However, RHO_2200 and RHO_0200 exhibit markedly different trends for frequencies greater than $1 \mathrm{~Hz}$.

Figure $12 \mathrm{~b}$ presents the variations of the correlation coefficients for CORR_2200, CORR_2200N, and CORR_0200 at the RW010 site. Regarding the "train noise" correlation coefficients, the CORR_2200 at the RW010 site presents a pattern similar to the one presented by the CORR_2200 at the RW002 site. The correlation coefficient of CORR_2200 is negative at low frequencies but become highly positive (between 0.8 and 1) when the frequencies are greater than $0.3 \mathrm{~Hz}$. The CORR_2200N and 

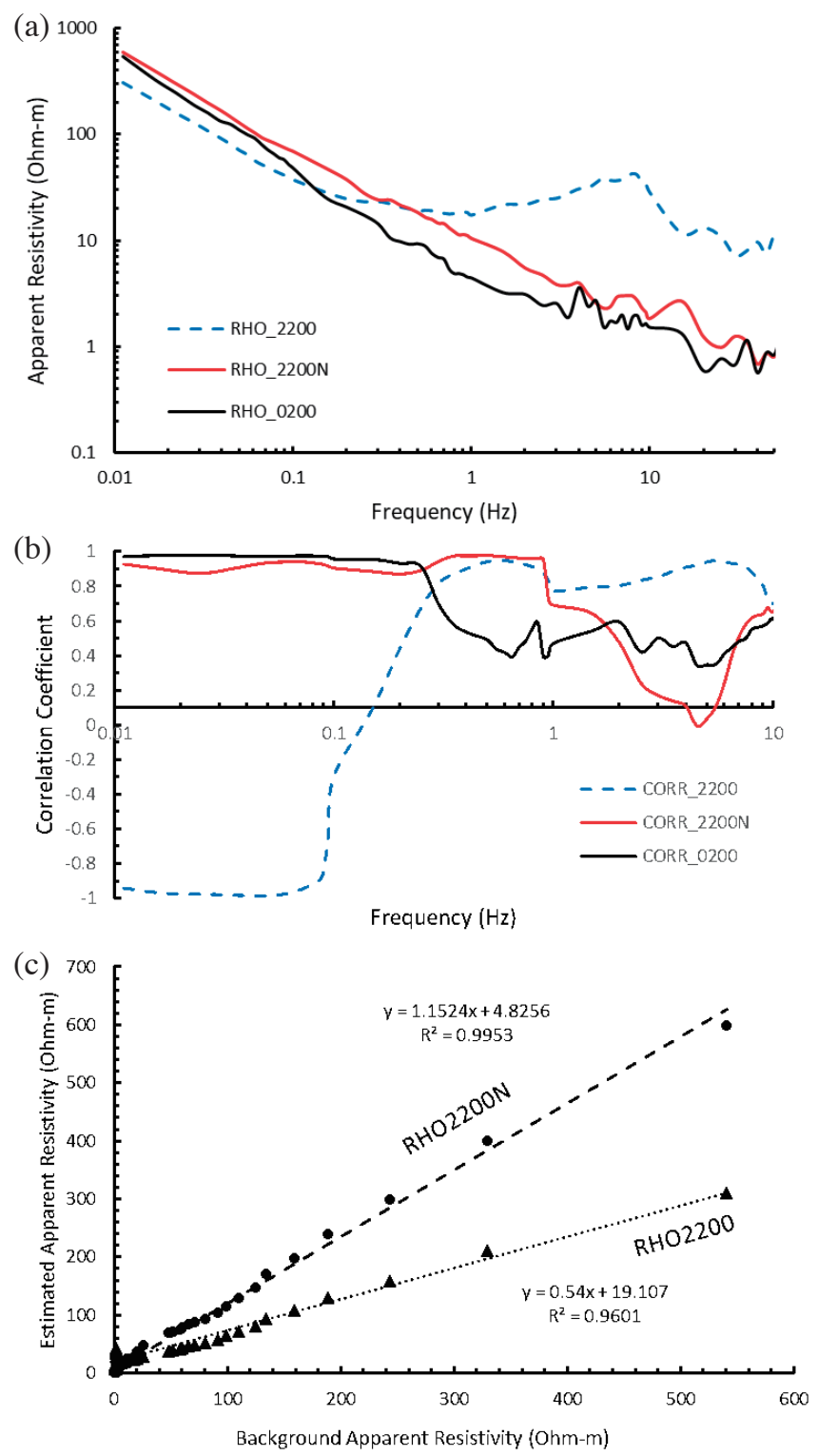

Fig. 12. (a) The RW010 apparent-resistivity measurements collected during the 22:00 - 22:59 period on 12/21/17 for both the "only trains passing" context (RHO_2200) and the "no trains passing" context (RHO_2200N), and the RW010 background-resistivity measurements (RHO_0200) collected during the 02:00 - 02:59 period on 12/22/17. (b) The correlation coefficients of the electric and magnetic spectra for the "only trains passing" context (CORR_2200) during the 22:00 - 22:59 period, the "no trains passing" context (CORR_2200N) during the 22:00 - 22:59 period, and the background context (CORR_0200) during the 02:00 - 02:59 period at RW002 on 12/22/17. (c) A comparison of the estimated apparent resistivities of RHO_2200 and RHO_2200N with the background resistivities of RHO_0200 at the RW010 site for frequencies less than 1Hz.

CORR_0200 are highly positive at frequencies lower than $0.3 \mathrm{~Hz}$. The correlation coefficient for CORR_2200N and CORR_0200 drop to lower than 0.8 at frequencies higher than 1 and $0.3 \mathrm{~Hz}$, respectively. Figure 12c compares the apparent resistivities of RHO_2200 and RHO_2200N to the apparent resistivity of the background (i.e., RHO_0200) for data with frequencies less than $1 \mathrm{~Hz}$. We compared the estimated resistivity of RHO_2200 and that of RHO_2200N with the background resistivity, and we observed that both of the estimated resistivities exhibit linear relationships with $r^{2}$ values of 0.96 and 0.99 , respectively (Fig. 10c). The ap- parent resistivity of RHO_2200 is about 1.1 times the background resistivity of RHO_0200 for frequencies less than 1 Hz. In addition, the apparent resistivity of RHO_2200N is about 56 percent of the apparent resistivity of RHO_0200 for frequencies less than $1 \mathrm{~Hz}$.

\section{CONCLUSIONS}

To study the possible influences that artificial electromagnetic signals from electric trains can have on magnetotelluric estimations, we conducted electromagnetic 
measurements near the tracks of the Taiwan railway system between the Luodong and Dongshan stations in Ilan. We collected the magnetotelluric signals at two sites, RW002 and RW010. Site RW002 is about $200 \mathrm{~m}$ from the train tracks, and site RW010 is about $1.1 \mathrm{~km}$ from the train tracks. From the time-series signals that we obtained, we identified the electric and magnetic signals corresponding to the timetable of electric trains. We discovered that the electric trains have the most influence (1) on the electric field in the direction parallel to the train track and (2) on the magnetic field in the direction perpendicular to the train track. In addition, our time-spectrum analysis, which covers both daytime signals and nighttime signals, shows that the noise frequencies of the train signals extended from $10^{-2}$ to over several decades measured in $\mathrm{Hz}$ even after applying the $60-\mathrm{Hz}$ notch filter. Thus we concluded that the notch filter cannot properly eliminated the noises induced by the train passing. We calculated the time-spectrum distribution by applying a 3-minute window, and then separated the signals affected by the train from those unaffected by the train during the 22:00 - 22:59 period. We also calculated the average power spectra for the measurements collected during the "quiet" early-morning period, extending from 02:00 to 02:59, and these spectra became our background data. We observed that the power spectra of the train-influenced magnetic field at the RW002 site are about two orders of magnitude larger than the corresponding power spectra at the RW010 for frequencies less than $1 \mathrm{~Hz}$. Furthermore, we observed that the difference between the power spectra at RW002 and RW010 gradually reduced to about a single order of magnitude in their high frequency components. By contrast, the power spectra of the train-influenced electric field is about the same as the power spectra of EX2200 at the RW010 site. These findings indicate that, regarding the distance from the observation sites to the railway tracks, there exists a train-associated magnetic field attenuated faster than the train-associated electric field. This fast-attenuated magnetic field may be affected by the magnetic dipole effect (Larsen et al. 1994) from currents flowing through overhead power lines and returning through the tracks. This magnetic dipole effect should be insignificant for stations that are more than $1 \mathrm{~km}$ from the railway. Our findings in this study show that the diffusion mechanism for train-derived EM noises differs from the diffusion mechanism for natural signals. Moreover, these train noises, if not excluded from measurements properly, may "contaminate" apparent-resistivitity estimations at low frequencies. We also concluded that random regional noises may strongly affect measurements for frequencies higher than $1 \mathrm{~Hz}$, since the correlation between natural electric fields and magnetic fields drops less than 0.8 at frequencies higher than $1 \mathrm{~Hz}$.

In addition, the apparent resistivities from "train passing" measurements and the apparent resistivities from "no train passing" measurements during the 22:00 - 22:59 period differ significantly from the "background" resistivities of the early-morning period at the RW002 site, although the first two sets of resistivities exhibit the same trends as the background resistivities when frequencies are less than $0.3 \mathrm{~Hz}$. By contrast, the apparent resistivities from "no trains passing" measurements during the 22:00 - 22:59 period are similar to the resistivities of the "background" resistivities during the early-morning period at the RW010 site. These facts, taken together, show that we can properly estimate apparent resistivity by excluding train noises from measurements at the RW010 site.

In our study, we did not examine the phase components of the train-contaminated and the noise-free magnetotelluric measurements. Our future work will focus on signals' phase components insofar as they may be affected by electric trains, including high-speed trains - a phenomenon that can distort data in subsurface-exploration research. Also, our future work will use forward modeling to examine different diffusion mechanisms of the railway electromagnetic signals, and to explore the possibility of using it as the energy sources for subsurface explorations.

Acknowledgements We are grateful to the field crewsMr. Yu-Chang Wu, Mr. Chia-Yu Sung, and Mr. Dong-Jin Jiang - for their help in preparing the fieldwork in NCU's near-surface geophysical lab. This project has been supported by Taiwan's Ministry of Science and Technology (Project \#106-2116-M-008-010).

\section{REFERENCES}

Acswoa, 2019: Taiwan's railway power system. Available at https://acswoa.tian.yam.com/posts/194804487. (in Chinese)

Cagniard, L., 1953: Basic theory of the magneto-telluric method of geophysical prospecting. Geophysics, 18, 605-635, doi: 10.1190/1.1437915. [Link]

Egbert, G. D., M. Eisel, O. S. Boyd, and H. F. Morrison, 2000: DC trains and Pc3s: Source effects in mid-latitude geomagnetic transfer functions. Geophys. Res. Lett., 27, 25-28, doi: 10.1029/1999g1008369. [Link]

Forbriger, T., 2007: Reducing magnetic field induced noise in broad-band seismic recordings. Geophys. J. Int., 169, 240-258, doi: 10.1111/j.1365-246x.2006.03295.x. [Link]

Fraser-Smith, A. C., 1981: Effects of man on geomagnetic activity and pulsations. Adv. Space Res., 1, 455-466, doi: 10.1016/0273-1177(81)90321-5. [Link]

Huang, S., B. Chen, Y. Kuo, K. Lu, and M. Huang, 2001: A short-circuit current study for the power supply system of Taiwan Railway. IEEE Power Engineering Review, 21, 61, doi: 10.1109/mper.2001.4311565. [Link]

Jones, F. W. and A. M. Kelly, 1966: Man-made telluric micropulsations. Can. J. Phys., 44, 3025-3031, doi: 10.1139/p66-247. [Link] 
Larsen, J., R. Mackie, T. Madden, A. Fiodelisi, A. Manzella, and S. Rieven, 1994: Robust processing for removing train signals from magnetotelluric data in central Italy. 12th Workshop on Electromagnetic Induction in the Earth, Brest, France.

Linington, R. E., 1974: The magnetic disturbances caused by DC electric railways. Prospezioni Archeologiche, 9, 9-20.

Lowes, F. J., 2009: DC railways and the magnetic fields they produce-The geomagnetic context. Earth Planets Space, 61, i-xv, doi: 10.1186/bf03352944. [Link]

Pádua, M. B., A. L. Padilha, and Í. Vitorello, 2002: Disturbances on magnetotelluric data due to DC electrified railway: A case study from southeastern Brazil. Earth Planets Space, 54, 591-596, doi: 10.1186/bf03353047.
[Link]

Simpson, F. and K. Bahr, 2005: Practical Magnetotellurics, Cambridge University Press, Cambridge, 254 pp, doi: 10.1017/CBO9780511614095. [Link]

Szarka, L., 1988: Geophysical aspects of man-made electromagnetic noise in the earth-A review. Surv. Geophys., 9, 287-318, doi: 10.1007/bf01901627. [Link]

Taiwan Railways Administration (TRA), 2017: Train timetable information, Taiwan Railways Administration, MOTC. Retrieved from http://twtraffic.tra.gov.tw/ twrail/EN QuickSearch.aspx.

Tanbo, T., H. Sakai, and T. Nagao, 2003: A study of geoelectric potential change caused by rail leak current observed at Ohtawa, Gifu, Japan. Electr. Eng. Jpn., 143, 1-10, doi: 10.1002/eej.10142. [Link] 